\title{
AN ALGORITHM FOR DETERMINING THE K-BEST SOLUTIONS OF THE ONE-DIMENSIONAL KNAPSACK PROBLEM
}

\author{
Horacio Hideki Yanasse \\ Instituto Nacional de Pesquisas Espaciais - \\ INPE/LAC \\ Nei Yoshihiro Soma \\ Instituto Tecnológico de Aeronáutica - ITA/IEC \\ Nelson Maculan \\ Universidade Federal do Rio de Janeiro - \\ UFRJ/COPPE
}

\begin{abstract}
In this work we present an enumerative scheme for determining the K-best solutions $(K>1)$ of the one dimensional knapsack problem. If $n$ is the total number of different items and $b$ is the knapsack's capacity, the computational complexity of the proposed scheme is bounded by $O(K n b)$ with memory requirements bounded by $O(n b)$. The algorithm was implemented in a workstation and computational tests for varying values of the parameters were performed.
\end{abstract}

Keywords: Knapsack problem, K-best solutions.

\section{Resumo}

Neste trabalho apresenta-se um esquema enumerativo para se determinar as K-melhores $(\mathrm{K}>1)$ soluções para o problema da mochila unidimensional. Se $n$ é o número total de itens diferentes e $b$ é a capacidade da mochila, a complexidade computacional do esquema proposto é limitado por $O(K n b)$. O algoritmo foi implementado em uma estação de trabalho e testes computacionais foram realizados variando-se diferentes parâmetros do problema.

Palavras-chave: Problema da mochila, K-melhores soluções. 


\section{INTRODUCTION}

Consider the one-dimensional knapsack problem (KP),

$$
\begin{aligned}
& \text { Maximize } \mathrm{z}=\sum_{i=1}^{n} \mathrm{c}_{\mathrm{i}} \mathrm{x}_{\mathrm{i}} \\
& \text { subject to } \quad \sum_{i=1}^{n} \mathrm{a}_{\mathrm{i}} \mathrm{x}_{\mathrm{i}} \leq \mathrm{b} \\
& \mathrm{x}_{\mathrm{i}} \geq 0 \quad \mathrm{i}=1,2, \ldots, \mathrm{n} \text { and integer }
\end{aligned}
$$

Our focus in this work is on the problem of finding the K-best solutions $(\mathrm{K}>1)$ for KP, instead of just a single optimal solution.

The KP is a well known NP-hard problem and is usually considered "well solved", since there are methods whose running time and space requirements are bounded by pseudo-polynomial functions in the input data, c.f. Toth [1980]; Yanasse and Soma [1987]; Soma, Yanasse, Zinober and Harley [1992]. Additionally, specialized branch-and-bound methods for solving the KP have a good performance in practice, c.f. Martello and Toth [1990].

Contrary to the case $\mathrm{K}=1$ where there is a vast literature (c.f. Martello and Toth [1990]), the case $\mathrm{K}>1$ is seldom addressed. We can point out the works by Lawler [1972] and Wolsey [1973], which do not specifically consider the KP but any discrete optimization problem, and more recently, the work of Yanasse and Soma [1990] which addresses the value independent knapsack problem.

Finding the K-best solutions of the Knapsack Problem (KKP) is of interest, for instance, when in addition to the knapsack constraint, there are some others which might be difficult to consider explicitly in a mathematical model, or if considered, would largely increase the size of the model. By finding the best, second best, ..., K-best solution, we are able to sequentially verify these solutions with respect to the additional constraints and stop when a solution that satisfies all of them is found. This situation usually appears in cutting stock problems - in addition to finding good combination of parts to be cut from a larger stock, cutting patterns must obey a series of constraints due to limitations of the cutting machine, material handling problems, order spread, etc.

Related to cutting, there are still other situations where the interest in finding the K-best solutions of a KP may arise. Cutting stock problems can be modeled as a set covering problem where each column represents a possible cutting pattern. In order to use such a formulation, we must generate at least some of the columns of the problem (the possible cutting patterns). The KP might appear as a subproblem for pattern generation, c.f. Gilmore and Gomory [1961, 1963, 1965], where each solution to the KP represents a cutting pattern. Generating a single pattern or several patterns at a time might be of interest, depending on the solution procedure adopted.

Another potential interest for solving the KKP arises in some approaches to integer problems. For instance, Maculan et al [1992] have proposed a column generation method to solve linear programming with bounding variable constraints, extending their results to the solution of integer problems. To solve some integer problems, their method requires a good implementation of an algorithm for the KKP.

Our interest in finding the K-best solutions is due to the difficulty we were faced with when we wanted to implement pseudo-polynomial algorithms for the one dimensional knapsack problem in small portable microcomputers. For relatively small values of $b$ and $n$, most DOS-based compilers already complain about memory overflow. One possible way to overcome this difficulty is to scale down the problem data. However, with the scaling operation we might loose optimality of 
the solutions determined. By checking the best $\mathrm{K}$ solutions of the scaled knapsack, we are able to verify them and make sure of getting the actual optimal solution for the original problem.

For the K-best KP, branch-and-bound methods do not perform as well as in the 1-best case since branches now cannot be fathomed, lower bounds for the K-best solution being hard to obtain. We are fairly confident that dynamic programming methods and/or other enumeration schemes for solving the K-best KP, are as competitive as any other method in terms of computational effort.

In the following session we propose an enumeration scheme for finding the K-best solutions to the KP. The enumeration scheme proposed allows a better implementation than the general algorithms suggested by Lawler [1972] and Wolsey [1973]. In section 3 we present computational tests using the proposed algorithm. Finally, section 4 contains some concluding remarks.

\section{The algorithm}

Without loss of generality, we assume from now on that the problem data is given in such a way that $\mathrm{a}_{1} \leq \mathrm{a}_{2} \leq \ldots \leq \mathrm{a}_{\mathrm{n}}$. For convenience, the KP with equality constraint and right hand side equal to $b$ is denoted by $\mathrm{KP}^{\mathrm{b}}$.

The proposed enumeration scheme is a recursive construction of optimal solutions to $\mathrm{KP}^{\mathrm{j}}$ for all possible values of the right hand side $\mathrm{j}$, from $\mathrm{a}_{1}$ up to $\mathrm{b}$. It follows closely the one presented in Yanasse and Soma [1987, 1990]. The method proposed can be viewed as an enumeration tree, where at each node we branch adding +1 to each one of the decision variables. So, for instance, at node $t$, we branch to get nodes $t_{1}, t_{2}, \ldots, t_{n}$, which are the result of adding 1 to $x_{j}, j=1, \ldots, n$ to the current solution at $\mathrm{t}$ (see Figure 1).

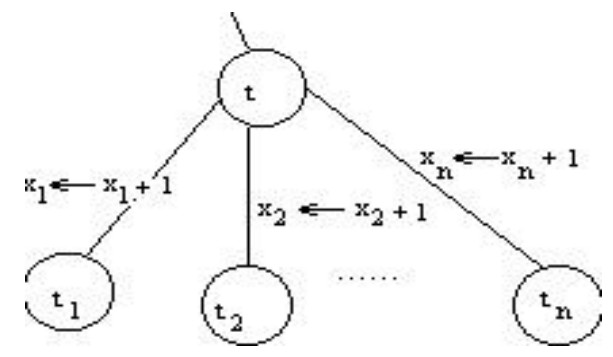

Figure 1

Branching scheme

Following a path from the initial node (node 0 ) to any node $t$ gives the values to be assigned to the decision variables in order to reach node $t$. Observe that adding in sequence, +1 to $x_{j}$, then +1 to $x_{k}, \ldots$, then +1 to $x_{S}$ or, by adding in sequence, +1 to $x_{j}$, then +1 to $x_{S}, \ldots$, then +1 to $x_{k}$ leads to the same solution. Hence, to avoid duplications, when branching from a node $t$, it is sufficient to consider nodes corresponding to adding +1 to decision variables with indices greater than or equal to the index of the incoming branch at node $t$.

Further savings in the enumeration process are obtained by grouping nodes $t$ and $m$ into a super node whenever the values of the knapsack constraint associated with these nodes are equal. This happens when $\mathrm{t}$ and $\mathrm{m}$ correspond to different combinations of the coefficients of the knapsack constraint leading to the same value. That is, if the decision variables in the knapsack constraint take the values obtained with the path from node zero to node $t$ and with the path from node 0 to node $\mathrm{m}$, the corresponding values are equal. 
Now, each super node $\mathrm{t}$ in the enumeration corresponds to the $\mathrm{KP}^{\mathrm{t}}$. And, we know beforehand that in our scheme we are interested in at most $\left(\mathrm{b}-\mathrm{a}_{1}\right)$ super nodes. From each super node $t$, we update $n-j+1$ new super nodes ("branching scheme"), where $j$ is the smallest index in super node $t$ for which we know there is a solution for $K P^{t}$, with $x_{j} \geq 1$. The super nodes to be updated are the ones determined by adding to $t$, the coefficient $a_{p}$, that is, super nodes $\left(t+a_{p}\right), p=j, j+1, \ldots, n$. Of course, only super nodes of interest are updated, that is super nodes $\left(t+a_{p}\right) \leq b$. Observe that the above operation corresponds to adding +1 to the variables with index $p, p=j, j+1, \ldots, n$. Indices smaller than $\mathrm{j}$ need not be considered since the corresponding combinations they produce are considered somewhere else. At each super node are kept the index $\mathrm{j}$ known to have at least one solution for $\mathrm{KP}^{\mathrm{t}}$ with $\mathrm{x}_{\mathrm{j}} \geq 1$, together with its corresponding best solution. Observe that the best solution value at a super node $\left(t+a_{p}\right)$, corresponding to index $p$, is obtained by adding to the best optimal solution values at super node $t$ corresponding to indices smaller than or equal to $p$, the coefficient $c_{p}, p=j, j+1, \ldots, n$. Starting with super node $t=0$, (the root super node, corresponding to $\left.\mathrm{KP}^{0}\right)$ with index set equal to $\{1,2, \ldots, \mathrm{n}\}$ and corresponding objective values $\{0,0, \ldots, 0\}$, the subsequent super nodes on which to "branch" are always chosen in increasing order of t. Only super nodes having at least one solution are considered. Once a super node $t>\left(b-a_{1}\right)$ is reached for branching, we stop. The forward enumeration part has been finished.

We illustrate the forward enumeration scheme by means of a numerical example. Consider the KP:

$$
\begin{array}{cl}
\text { Maximize } & \mathrm{z}=4 \mathrm{x}_{1}+3 \mathrm{x}_{2}+5 \mathrm{x}_{3}+7 \mathrm{x}_{4}+8 \mathrm{x}_{5} \\
\text { subject to } & 3 \mathrm{x}_{1}+4 \mathrm{x}_{2}+5 \mathrm{x}_{3}+6 \mathrm{x}_{4}+7 \mathrm{x}_{5} \leq 15 \\
& \mathrm{x}_{\mathrm{i}} \geq 0 \quad \mathrm{i}=1,2, \ldots, 5 \text { and integer. }
\end{array}
$$

Initially, we start with super node 0 , with an attached set of indices $\{1,2,3,4,5\}$ and corresponding values of the objective function $\{0,0,0,0,0\}$. Nodes $3,4, \ldots, 15$ are the other super nodes whose set of indices and corresponding objective function values are to be updated. Observe that super nodes 1 and 2 (all super nodes $\mathrm{t}<\mathrm{a}_{1}$ ) are never updated since they cannot be reached from super node 0 (that is, there is no solution with right hand sides smaller than $\mathrm{a}_{1}$ ). Since the smaller index in the index set attached to super node 0 is 1 , from super node 0 , the following super nodes are to be updated: $\left(0+\mathrm{a}_{1}\right)=3,\left(0+\mathrm{a}_{2}\right)=4,\left(0+\mathrm{a}_{3}\right)=5,\left(0+\mathrm{a}_{4}\right)=6,\left(0+\mathrm{a}_{5}\right)=7$. The corresponding objective function values are $(0+4),(\max \{0,0\}+3),(\max \{0,0,0\}+5),(\max \{0,0,0,0\}+7)$, $(\max \{0,0,0,0,0\}+8)$, respectively. We represent this information in Table 1 .

In Table 1, columns 1 to 5 give indication of the index attached to a super node. The super nodes are indicated in the last column. The numbers filled in the table in any column $\mathrm{j}$ are the best objective values corresponding to a feasible solution having at least $x_{j} \geq 1$. For instance, column 4 , node 6 , has a value 7 , indicating that there is a solution to $\mathrm{KP}^{6}$ with $\mathrm{x}_{4} \geq 1$ and corresponding objective function value equal to 7 .

The subsequent super node to branch is super node 3 , which is the next super node after 0 that has at least one solution. Since the smaller index in the index set attached to super node 3 is 1 again, from super node 3 , the following super nodes are to be updated: $\left(3+a_{1}\right)=6,\left(3+a_{2}\right)=7$, $\left(3+a_{3}\right)=8,\left(3+a_{4}\right)=9,\left(3+a_{5}\right)=10$. The corresponding objective function values are $(4+4)$, $(4+3),(4+5),(4+7),(4+8)$, respectively. This information is shown in Table 2.

Observe now in Table 2, that there are some rows (super nodes) with more than 2 objective function values. This indicates a multiplicity of feasible solutions for the knapsack problem corresponding to that super node. For instance, from row 6 , we can conclude that $\mathrm{KP}^{6}$ has at least 2 solutions with objective values 8 and 7 and one of the solutions has $x_{1} \geq 1$ and the other $x_{4} \geq 1$. 
Table 1

Initial updating from super node 0

\begin{tabular}{|c|c|c|c|c||c||}
\hline \multicolumn{5}{||c|}{ Index } & \multicolumn{1}{c|}{} \\
\hline \hline 1 & 2 & 3 & 4 & 5 & SN \\
\hline \hline $\mathbf{0}$ & $\mathbf{0}$ & $\mathbf{0}$ & $\mathbf{0}$ & $\mathbf{0}$ & $\underline{\boldsymbol{0}}$ \\
\hline $\mathrm{X}$ & $\mathrm{X}$ & $\mathrm{X}$ & $\mathrm{X}$ & $\mathrm{X}$ & 1 \\
\hline $\mathrm{X}$ & $\mathrm{X}$ & $\mathrm{X}$ & $\mathrm{X}$ & $\mathrm{X}$ & 2 \\
\hline $\mathbf{4}$ & & & & & 3 \\
\hline & $\mathbf{3}$ & & & & 4 \\
\hline & & $\mathbf{5}$ & & & 5 \\
\hline & & & $\mathbf{7}$ & & 6 \\
\hline \hline & & & & $\mathbf{8}$ & 7 \\
\hline & & & & & 8 \\
\hline & & & & & 9 \\
\hline & & & & & 10 \\
\hline & & & & & 11 \\
\hline & & & & & 12 \\
\hline & & & & & 13 \\
\hline & & & & & 14 \\
\hline & & & & & 15 \\
\hline
\end{tabular}

Table 2

Updating from super node 3

\begin{tabular}{|c|c|c|c|c|c|}
\hline \multicolumn{5}{|c|}{ Index } & \multirow[b]{2}{*}{ SN } \\
\hline 1 & 2 & 3 & 4 & 5 & \\
\hline 0 & 0 & 0 & 0 & 0 & 0 \\
\hline $\mathrm{X}$ & $\mathrm{X}$ & $X$ & $\mathrm{X}$ & $X$ & 1 \\
\hline$X$ & $X$ & $X$ & $X$ & $X$ & 2 \\
\hline \multirow[t]{3}{*}{4} & $X$ & $X$ & $X$ & $X$ & $\underline{3}$ \\
\hline & 3 & & & & 4 \\
\hline & & 5 & & & 5 \\
\hline \multirow[t]{10}{*}{8} & & & 7 & & 6 \\
\hline & 7 & & & 8 & 7 \\
\hline & & 9 & & & 8 \\
\hline & & & 11 & & 9 \\
\hline & & & & 12 & 10 \\
\hline & & & & & 11 \\
\hline & & & & & 12 \\
\hline & & & & & 13 \\
\hline & & & & & 14 \\
\hline & & & & & 15 \\
\hline
\end{tabular}

The subsequent super node to branch is super node 4 , then, super node 5 and the updating is performed as before. We discuss further the branching of super node 6 , which presents new particularities. The smaller index in the index set attached to super node 6 is 1 , hence, from super node 6 , the following super nodes are to be updated: $\left(6+a_{1}\right)=9,\left(6+a_{2}\right)=10,\left(6+a_{3}\right)=11,\left(6+a_{4}\right)=12$, $\left(6+a_{5}\right)=13$. The corresponding objective function values are $(8+4),(8+3),(8+5),(\max \{8,7\}+7)$, $(\max \{8,7\}+8)$, respectively. Observe that super nodes 12 and 13 are updated only with the best objective function values 15 and 16, respectively, but there is an alternative solution in each one of these super nodes, with corresponding objective values of 14 and 15, respectively. We present the result of the enumeration made up to this point in Table 3 and in Table 4, the final result of the enumeration process is displayed. 
Table 3

Updated table after branching from super node 6

\begin{tabular}{||c|c|c|c|c||c||}
\hline \multicolumn{7}{|c|}{ Index } & \multicolumn{1}{c||}{} \\
\hline $\mathrm{1}$ & 2 & 3 & 4 & 5 & SN \\
\hline 0 & 0 & 0 & 0 & 0 & 0 \\
\hline $\mathrm{X}$ & $\mathrm{X}$ & $\mathrm{X}$ & $\mathrm{X}$ & $\mathrm{X}$ & 1 \\
\hline $\mathrm{X}$ & $\mathrm{X}$ & $\mathrm{X}$ & $\mathrm{X}$ & $\mathrm{X}$ & 2 \\
\hline 4 & $\mathrm{X}$ & $\mathrm{X}$ & $\mathrm{X}$ & $\mathrm{X}$ & 3 \\
\hline $\mathrm{X}$ & 3 & $\mathrm{X}$ & $\mathrm{X}$ & $\mathrm{X}$ & 4 \\
\hline $\mathrm{X}$ & $\mathrm{X}$ & 5 & $\mathrm{X}$ & $\mathrm{X}$ & 5 \\
\hline $\mathbf{8}$ & $\mathrm{X}$ & $\mathrm{X}$ & 7 & $\mathrm{X}$ & $\mathbf{6}$ \\
\hline \hline & 7 & & & 8 & 7 \\
\hline & 6 & 9 & & & 8 \\
\hline $\mathbf{1 2}$ & & 8 & 11 & & 9 \\
\hline & $\mathbf{1 1}$ & & 10 & 12 & 10 \\
\hline & & $\mathbf{1 3}$ & & 11 & 11 \\
\hline & & & $\mathbf{1 5}$ & & 12 \\
\hline & & & & $\mathbf{1 6}$ & 13 \\
\hline & & & & & 14 \\
\hline & & & & & 15 \\
\hline
\end{tabular}

Table 4

Table obtained at the end of the forward enumeration

\begin{tabular}{|c|c|c|c|c||c||}
\hline \multicolumn{7}{|c|}{ Index } \\
\hline 1 & 2 & 3 & 4 & 5 & SN \\
\hline \hline 0 & 0 & 0 & 0 & 0 & 0 \\
\hline$X$ & $X$ & $X$ & $X$ & $X$ & 1 \\
\hline$X$ & $X$ & $X$ & $X$ & $X$ & 2 \\
\hline 4 & $X$ & $X$ & $X$ & $X$ & 3 \\
\hline$X$ & 3 & $X$ & $X$ & $X$ & 4 \\
\hline$X$ & $X$ & 5 & $X$ & $X$ & 5 \\
\hline 8 & $X$ & $X$ & 7 & $X$ & 6 \\
\hline$X$ & 7 & $X$ & $X$ & 8 & 7 \\
\hline$X$ & 6 & 9 & $X$ & $X$ & 8 \\
\hline 12 & $X$ & 8 & 11 & $X$ & 9 \\
\hline$X$ & 11 & 10 & 10 & 12 & 10 \\
\hline$X$ & 10 & 13 & 12 & 11 & 11 \\
\hline 16 & 9 & 12 & 15 & 13 & 12 \\
\hline & 15 & 14 & 14 & 16 & 13 \\
\hline & 14 & 17 & 16 & 16 & 14 \\
\hline 20 & 13 & 16 & 19 & 17 & 15 \\
\hline \multicolumn{7}{|c|}{}
\end{tabular}

Observe that we can easily determine a feasible solution to KP corresponding to a specified objective function value using the information in the Table. Consider, for instance, the objective function value 19, in super node 15 . The immediate information we have from the table is that there is a solution to $\mathrm{KP}^{15}$, with objective value 19 and with $\mathrm{x}_{4} \geq 1$. Since $\mathrm{x}_{4} \geq 1$, we subtract $\mathrm{a}_{4}$ from 15 , leading to the right hand side 9 and the corresponding objective value of 12, which is determined by subtracting $\mathrm{c}_{4}$ from 19 . So, there should be a solution for $\mathrm{KP}^{9}$ with objective value 12 and, due to the forward enumeration process, we also know that this value should be located in the table in some column corresponding to an index smaller than or equal to 4 . In fact at super node 9 , the value 12 can be found in column 1. The same reasoning is applied again. There is a solution to $\mathrm{KP}^{9}$, with objective value 12 and with $\mathrm{x}_{1} \geq 1$. Since $\mathrm{x}_{1} \geq 1$, we subtract $\mathrm{a}_{1}$ from 9 , leading to the new right hand side 6 and the corresponding objective value of 8 , which is determined by subtracting $\mathrm{c}_{1}$ from 12 . So, there should be a solution for $\mathrm{KP}^{6}$ with objective value 8 and we will find it in the table in some column corresponding to an index smaller than or equal to 1 . This is verified and the same reasoning is applied again and again, until we finally reach node 0 . At this stage we have determined a solution to the equality constrained KP with right hand side 15, and objective function value equal to 19 , which in our case would be: $x_{1}=3, x_{4}=1, x_{2}=x_{3}=x_{5}=0$. 


\section{Recovering the K-best solutions}

To recover the K-best solutions a list of the K-best objective function values to the KP is built by traversing the super nodes from $b, b-1, \ldots, 0$, and by choosing the K-best solution values. Unfortunately, they are not always the K-best solutions to our KP. There might be alternative solutions with the same objective function value or a little bit smaller which are not explicitly indicated in the final table. It is necessary, therefore, to check the existence of any other alternative solution better than the $\mathrm{K}^{\text {th }}$ element listed so far. The only thing that can be stated at this point is that the $\mathrm{K}^{\text {th }}$ element in the list is just a lower bound for the K-best optimal solution value of the KP.

In our previous numerical example, let us assume $\mathrm{K}=15$. The initial list containing the best $\mathrm{K}$ (=15) objective function values to the KP are: $20,19,17,17,16,16,16,16,16,15,15,14,14$, 14 and 13 . Hence, a lower bound on the $15^{\text {th }}$-best objective function value is 13 and a check verifying whether there are other solutions, not explicitly indicated in the final enumeration table, better than the $\mathrm{K}^{\text {th }}$ solution, is, then, performed.

The recovering of the K-best solutions is achieved by analyzing the solutions corresponding to the best $\mathrm{K}$ values already identified in the list. In the process, we search for alternative solutions having objective values better than the $\mathrm{K}^{\text {th }}$ best value identified so far. Our search will start analyzing the best, second best, third best and so on solutions, hoping that by analyzing first the best solutions, there would be an increased chance of finding alternative solutions as good as the ones being considered. Once the analysis of a $\mathrm{J}^{\text {th }}$-best solution with value equal to the $\mathrm{K}^{\text {th }}$ best in the list is reached we can stop. The K-best solution values are now at hand and it is necessary only to determine the remaining solutions corresponding to the objective values in the list, which were not considered yet.

Alternative solutions are identified in the process of recovering a solution to a specific objective value, whenever more than one feasible solution is observed in any intermediate super node during backtracking. For instance, in the previous recovering process example with super node 15 and objective value 19, a backtrack in super node 9 detected 3 solutions with objective values $(12,8,11)$ identified in the table. All three of them are feasible in the sense that super node $15 \mathrm{can}$ be reached from them and moreover, a numerical value in column 4 indicates that all these values are in columns with indices smaller than or equal to 4 . Hence, with all of them, by adding +1 to variable $\mathrm{x}_{4}$, leads to super node 15 , column 4 . In this example, the objective values corresponding to these alternative solutions are different: 19,15 and 18, respectively. Only one of the solutions reaches the value 19 , the best value with at least one $\mathrm{x}_{4} \geq 1$.

The full recovery of the 15-best solutions of our numerical example is illustrated now. The process starts with the 1-best solution value 20. Backtracking, it is seen that the value 20 in super node 15 came by adding +1 to variable $\mathrm{x}_{1}$. Subtracting $\mathrm{a}_{1}$ from 15 yields super node 12 , with corresponding objective value of $16=20-\mathrm{c}_{1}$.

We are again in a similar situation and proceed in the same way, concluding that there is no alternative solution for this case. The single solution $\mathrm{x}_{1}=5, \mathrm{x}_{2}=\mathrm{x}_{3}=\mathrm{x}_{4}=\mathrm{x}_{5}=0$, with objective function value 20 has been obtained.

The next solution to be considered is the second best one, whose objective function value is 19 . As previously pointed out, there are alternative solutions in this case. Recall that with the backtracking from super node 15 , solution value 19, super node 9 was reached with alternative solutions. In the process of analyzing these alternative solutions, we start with the largest index smaller than or equal to 4 . A solution of value 11 is found at column 4 in row 9 , indicating that a solution exists with corresponding objective function value of 18 . This value is compared with the current $15^{\text {th }}$-best value in the list to see if this solution will be included among the 15 -best or is to be discarded. In this case, a better solution has been obtained, hence, this value is introduced in the list and the previous $15^{\text {th }}$ value is deleted. With the new list, the lower bound for the $15^{\text {th }}$ best solution is updated and the process is repeated from where it has been previously halted, i.e. 
from super node 9 , column 4 and objective value 11 . We backtrack subtracting a from 9 . This gives super node 3 with corresponding objective value 4 . There is a single solution in node 3 and proceeding with the backtracking up to super node 0 a solution is identified, given by $\mathrm{x}_{1}=1, \mathrm{x}_{4}$ $=2, x_{2}=x_{3}=x_{5}=0$, with corresponding objective value 18 .

Then we return to the smallest super node where an alternative solution was identified, super node 9 in this case. It is necessary now to move to the immediate next column with a smaller index with a solution, that is column 3. A solution of value 8 is found, indicating that a solution exists with corresponding objective function value of 15 . Again, this value is compared with the $15^{\text {th }}$-best value in the list, and since this latter one is greater than the former, it is, therefore, introduced in the list and the previous $15^{\text {th }}$ value is discarded. The lower bound for the $15^{\text {th }}$-best solution is updated and the process is re-launched from where it has stopped.

Since the last super node considered was the 9 , column 3 , with objective value 8 a backtracking is performed by subtracting a 3 from 9 , reaching super node 4 with corresponding objective value of 3 . There is just a single solution in super node 4 and proceeding with the backtracking up to super node 0 the solution $\mathrm{x}_{2}=1, \mathrm{x}_{3}=1, \mathrm{x}_{4}=1, \mathrm{x}_{1}=\mathrm{x}_{5}=0$, with corresponding objective value 15 is identified.

The smallest super node where an alternative solution was identified, i.e. super node 9 is considered again and the immediate next column, which has yet to be considered, with a smaller index possessing a solution is column 1. A solution of value 12 is found, and after a backtracking no more alternative solutions are found, and the single solution $\mathrm{x}_{1}=3, \mathrm{x}_{4}=1, \mathrm{x}_{2}=\mathrm{x}_{3}=\mathrm{x}_{5}=0$, with corresponding objective function value of 19 is identified.

We move now to the next best value in the list not yet analyzed. At this stage, the list has changed to $20^{*}, 19^{*}, 18^{*}, 17,17,16,16,16,16,16,15^{*}, 15,15,14,14$, where the values indicated with an * are the ones which have already been analyzed (the corresponding solutions have already been obtained). The third best value in the list is 18 and it was considered during the analysis of the $2^{\text {nd }}$ best value. Hence, the $4^{\text {th }}$-best value, which is 17 , is considered next.

Proceeding with the same reasoning, we are in super node 15 , column 5 , objective value 17 , and the backtracking implies in subtracting a5 from 15, reaching super node 8 which has two solutions. Starting again the recovering process with the largest index smaller than or equal to 5 , a solution of value 9 is found at column 3 in row 8 , indicating a solution with corresponding objective function value of 17 . We backtrack subtracting a from 8 to reach super node 3 with corresponding objective value 4 . There is a single solution in node 3 and proceeding with the backtracking up to super node 0 the solution $\mathrm{x}_{1}=1, \mathrm{x}_{3}=1, \mathrm{x}_{5}=1, \mathrm{x}_{2}=\mathrm{x}_{4}=0$ is identified, with corresponding objective value 17 .

Returning to the smallest super node where an alternative solution was identified, super node 8 , we move to the immediate next column with a smaller index with a solution, that is column 2. A solution of value 6 is found indicating a solution with corresponding objective function of 14 . Since this is worse than the $15^{\text {th }}$-best value in the current list, this solution is discarded. No more alternative solution are present, we then move to the $5^{\text {th }}$-best value of the list, which is again 17 .

This is in super node 14, column 3 , objective value 17 , and a backtracking is carried out by subtracting a 3 from 14 reaching super node 9. In this super node there are 3 solutions,. however, only two of them with indices smaller than or equal to 3 . Starting with the largest index smaller than or equal to 3 , a solution of value 8 is found at column 3 in row 9, indicating a solution with corresponding objective function of 13 . Since this is worse than the $15^{\text {th }}$ best current value, this solution is disregarded and the next alternative solution is examined. Moving to the immediate next column with a smaller index and with a solution, that is column 1, a solution of value 12 is found indicating a solution with corresponding objective function of 17. Since this solution is better than the 15 th best in the list, we proceed with the backtracking, arriving at the solution $\mathrm{x}_{1}$ 
$=3, x_{3}=1, x_{2}=x_{4}=x_{5}=0$, with corresponding objective value 17 . No more alternative solutions are present, so the $6^{\text {th }}$-best value of the list, which is 16 , is examined next.

Proceeding with this analysis, a stage is reached when the 15th-best value is 15 and there are only values 15 in the list to be analyzed. In this case, we are sure that the 15 th best value in the list is the optimal value of the 15-best solution of the KP and hence, further searches for alternative solutions are not necessary; what is left is just the recovering of the solution corresponding to each one of the remaining values of the list.

The 15-best solutions to our numerical example are presented in Table 5.

Table 5

15-best solutions to the example

\begin{tabular}{|c||c|c||c||c||c||}
\hline \multicolumn{7}{|c||}{ Index } \\
\hline \hline 1 & 2 & 3 & 4 & 5 & OV \\
\hline \hline 5 & 0 & 0 & 0 & 0 & 20 \\
\hline 3 & 0 & 0 & 1 & 0 & 19 \\
\hline 1 & 0 & 0 & 2 & 0 & 18 \\
\hline 1 & 0 & 1 & 0 & 1 & 17 \\
\hline 3 & 0 & 1 & 0 & 0 & 17 \\
\hline 2 & 1 & 1 & 0 & 0 & 16 \\
\hline 0 & 0 & 0 & 0 & 2 & 16 \\
\hline 1 & 0 & 1 & 1 & 0 & 16 \\
\hline 2 & 0 & 0 & 0 & 1 & 16 \\
\hline 4 & 0 & 0 & 0 & 0 & 16 \\
\hline 0 & 1 & 1 & 1 & 0 & 15 \\
\hline 0 & 0 & 3 & 0 & 0 & 15 \\
\hline 1 & 1 & 0 & 0 & 1 & 15 \\
\hline 0 & 0 & 0 & 1 & 1 & 15 \\
\hline 3 & 1 & 0 & 0 & 0 & 15 \\
\hline \hline
\end{tabular}

As can be seen, the memory requirements for the proposed enumeration scheme is $\mathrm{O}\left(\mathrm{n}\left(\mathrm{b}-\mathrm{a}_{1}\right)\right)$. In fact, if $\mathrm{K}<\mathrm{n}$, we need to keep only $\mathrm{K}$ values for every super node $t$ corresponding to $K \mathrm{P}^{\mathrm{t}}, \mathrm{t}=$ $\mathrm{a}_{1}, \ldots, \mathrm{b}$. Hence, memory requirements for the algorithm is $\mathrm{O}\left(\min \{\mathrm{K}, \mathrm{n}\}\left(\mathrm{b}-\mathrm{a}_{1}\right)\right)$, and in particular, for $\mathrm{K}=1, \mathrm{O}\left(\mathrm{b}-\mathrm{a}_{1}\right)$. The computational complexity of the algorithm is bounded by $\mathrm{O}((\mathrm{K}+1) \mathrm{n}(\mathrm{b}-$ $\left.\mathrm{a}_{1}\right)$ ), since the forward enumeration is bounded by $\mathrm{O}\left(\mathrm{n}\left(\mathrm{b}-\mathrm{a}_{1}\right)\right)$ and to recover the $\mathrm{K}$ solutions (any $\mathrm{K}$ ), we surely require no more than $\mathrm{O}\left(\mathrm{K} \min \{\mathrm{K}, \mathrm{n}\}\left(\mathrm{b}-\mathrm{a}_{1}\right)\right)$ operations (we, at worse, must backtrack this much of the forward enumeration performed, for each one of the solutions). Generally, recovering each solution is quite straightforward taking $\mathrm{O}\left(\left(\mathrm{b}-\mathrm{a}_{1}\right) / \mathrm{a}_{1}\right)$ operations, hence, the recovery time for the K-best solutions will generally be smaller compared with the forward enumeration time. This was observed with the computational results presented in the next section.

\section{Computational experiments}

We performed some computational tests to observe the effect on computational time of varying values of the right hand side $\mathrm{b}$, the number of variables $\mathrm{n}$, and the number, $\mathrm{K}$, of best solutions desired. For each set of parameters tested, a fixed sample size of 20 was used. The test problems 
were constructed artificially with all coefficients $a_{j}$ of the knapsack constraint generated randomly in the interval $[1,5000]$.

Our proposed enumeration scheme was implemented in C++ on an IBM RISC/6000, Model $580 \mathrm{H}$, with an IBM Power PC 2 processor and 256 Mbytes of RAM memory. All processing times were measured in seconds.

For the first set of tests, we varied the number of the K-best solutions required. K was set to 100 , $200, \cdots, 2800,2900$. b was set to 10000 and $\mathrm{n}$ to 500 . Figure 2 presents the results.

$n=500, b=10000$

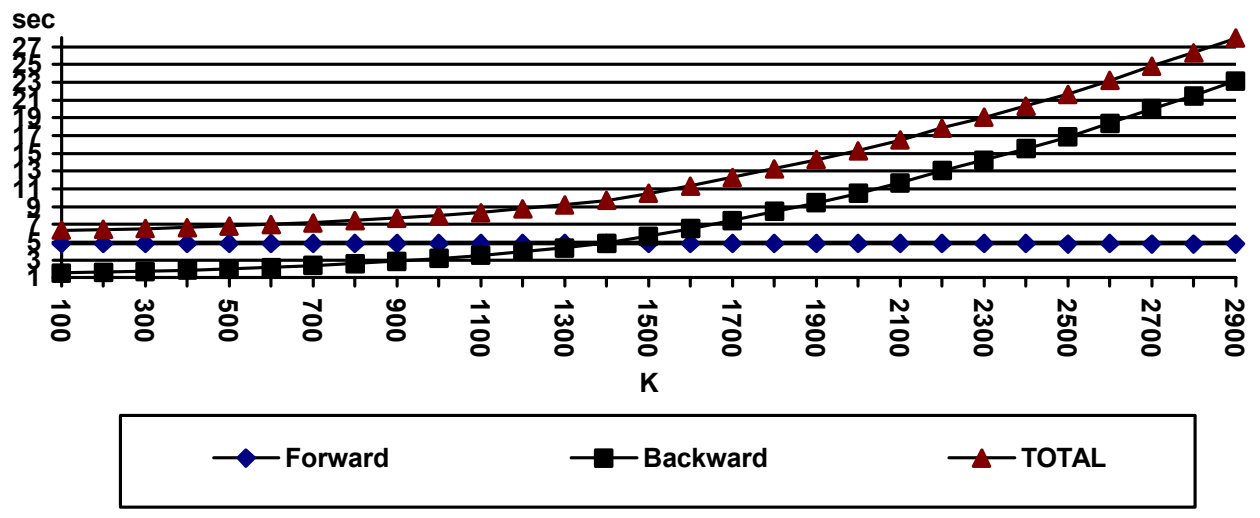

Figure 2

Computational times with varying $\mathrm{K}$

In the second set of problems we varied the number of variables $n$. $n$ varied from 100 to 1050 in steps of $50 . \mathrm{K}$ and $\mathrm{b}$ were fixed respectively to 1000 and 10000 . The results are shown in Figure 3.

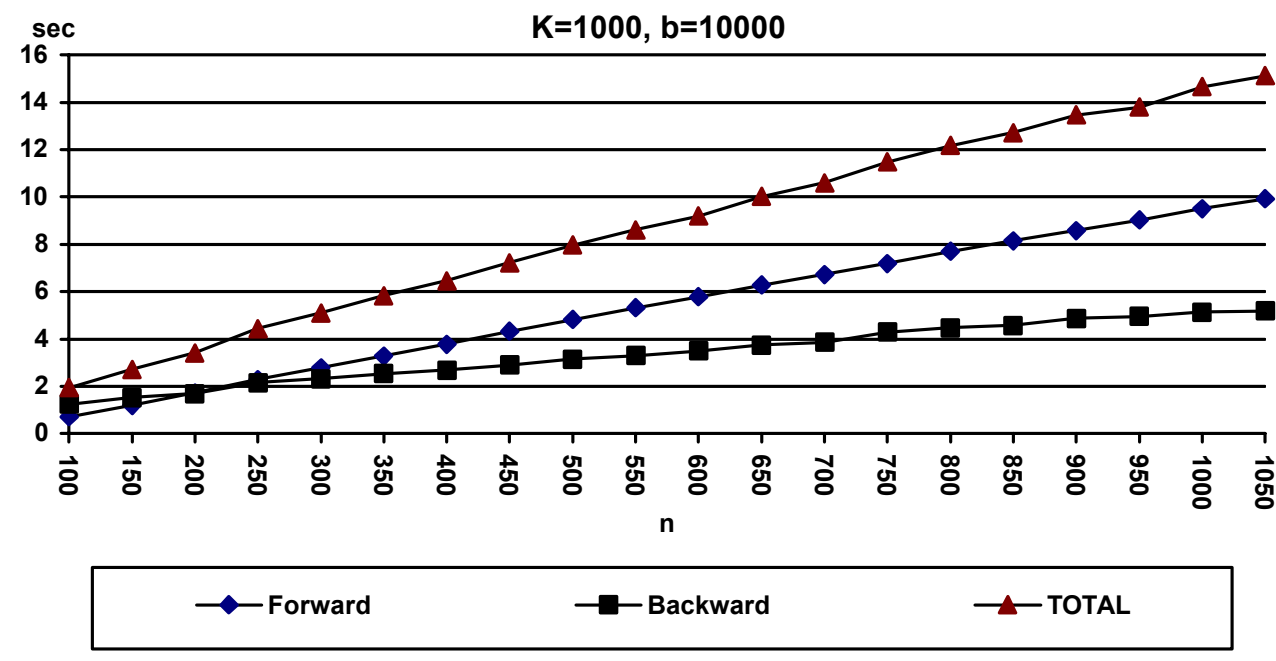

Figure 3

Effect of varying $n$. 
In the last set of tests we varied the value of $b$ from 5000 to 16000 , in steps of 1000 . The number of variables and $\mathrm{K}$ were fixed in 500 and 1000, respectively. The results are presented in Figure 4.

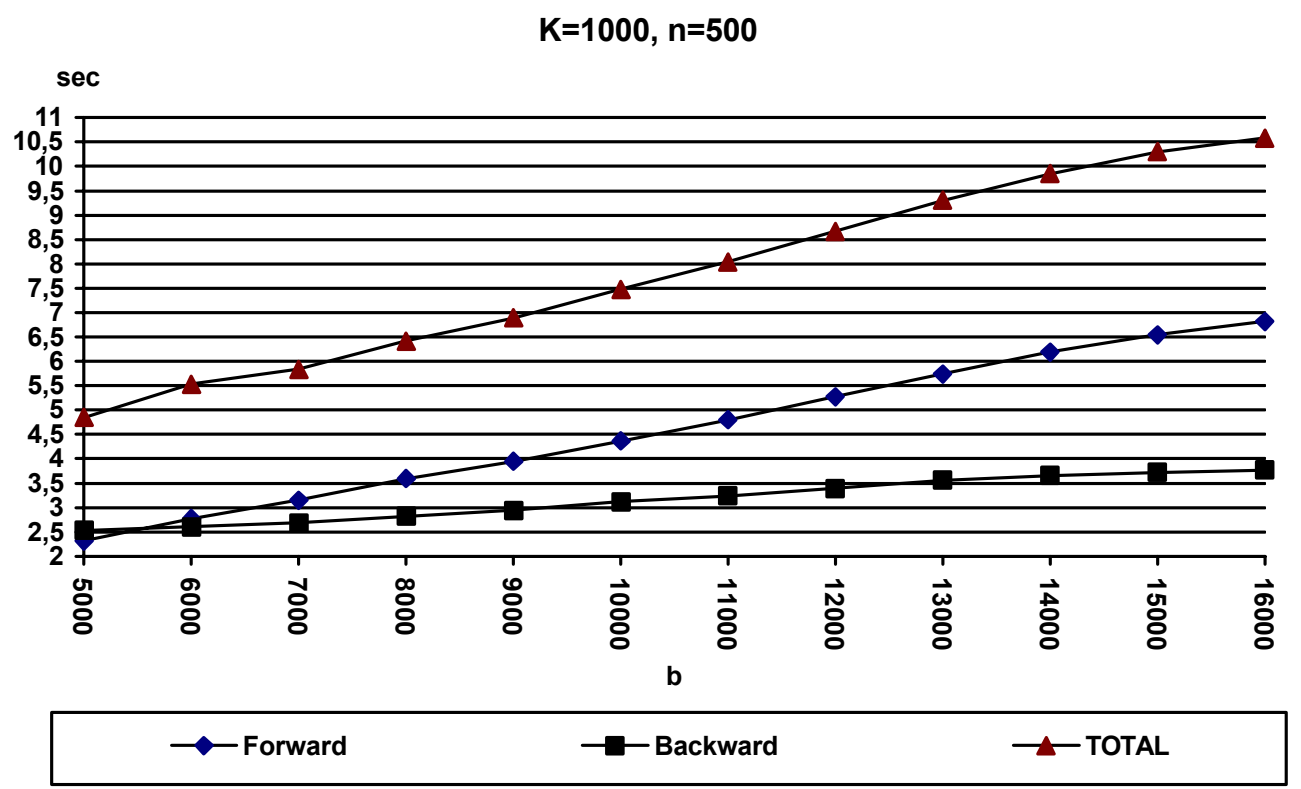

Figure 4

Effect of varying $b$

The forward enumeration becomes responsible for most of the processing time, as $\mathrm{n}$ increases. Retrieving the solutions demands an amount of time which depends on $\mathrm{K}$ and $\mathrm{n}$, but we observed that it is quite insensitive with respect to variations in the value of $b$ (perhaps because of the small variations of this parameter for the tests performed). Its contribution to the overall computer processing time sharply decreases (percentagewise) as the size of the problem increases. For $\mathrm{K}<\mathrm{n}$, the time complexity of the recovery phase should vary as $\mathrm{K}^{2} \mathrm{~b}$ and for $\mathrm{K}>\mathrm{n}$ as $\mathrm{Knb}$. The backward curve in Figure 2 suggests a quadratic behaviour with K, even for K larger than $\mathrm{n}$. A possible explanation for this behaviour is due to the fact that all the tests were run using the same implemented version of the algorithm where the number of columns was maintained fixed. Recall that in case of $\mathrm{K}<\mathrm{n}$, only $\mathrm{K}$ columns are needed to be carried on in the forward enumeration. Another explanation is the computational time spent to sort the solutions. Recovered solutions are sorted as they are obtained, however, no special care was taken to use an efficient sorting routine in the implemented version of the algorithm. Therefore, the curves in Figure 2 are overestimating the actual ones that would have been obtained with an improved implementation.

\section{Concluding remarks}

As stated previously, we were unable to obtain other references on the KKP. The general methods proposed by Lawler [1972] and Wolsey [1973] can be applied to the KP but the implementation proposed here is better. Lawler's method has a computational complexity of $\mathrm{O}\left(\mathrm{Kn}^{2}\left(\mathrm{~b}-\mathrm{a}_{1}\right)\right)$ and the computational complexity of Wolsey's method is hard to determine since it uses a series of branch-and-bound problems. We have yet to perform computational tests comparing all these methods. 
The proposed enumeration scheme can be easily extended to solve knapsack problems with bounded variables, in particular the 0-1 case. Notice that the equality constrained KP can also be easily solved. In fact it was solved as a by-product of our proposed enumeration scheme.

Improved computational time performance might be obtained if additional information are kept. For instance, we can keep in another array, the index and the best value for each super node. Hence, these optimal solutions for each KPt, will be ready for consulting when needed, otherwise, every time we need such information at each super node, it is necessary to make a search among $n$ elements.

It is of course possible to have an implementation of the algorithm with a reduced memory requirement. The authors can propose an implementation using $\mathrm{O}\left(\mathrm{b}-\mathrm{a}_{1}\right)$ of memory space but the computational effort to recover the solutions will be much increased.

We would like to observe that the proposed enumeration scheme presented is just the KP solved as a longest path problem, where we are interested in finding the longest path from node 0 to node $b$. The "distance" from a node $j$ to a node $j+a_{k}$ in the "knapsack graph" is given by $c_{k}$, for each one of the nodes $j=0,1,2, \ldots, b$, and for every $k=1,2, \ldots, n$ (see Figure 5).

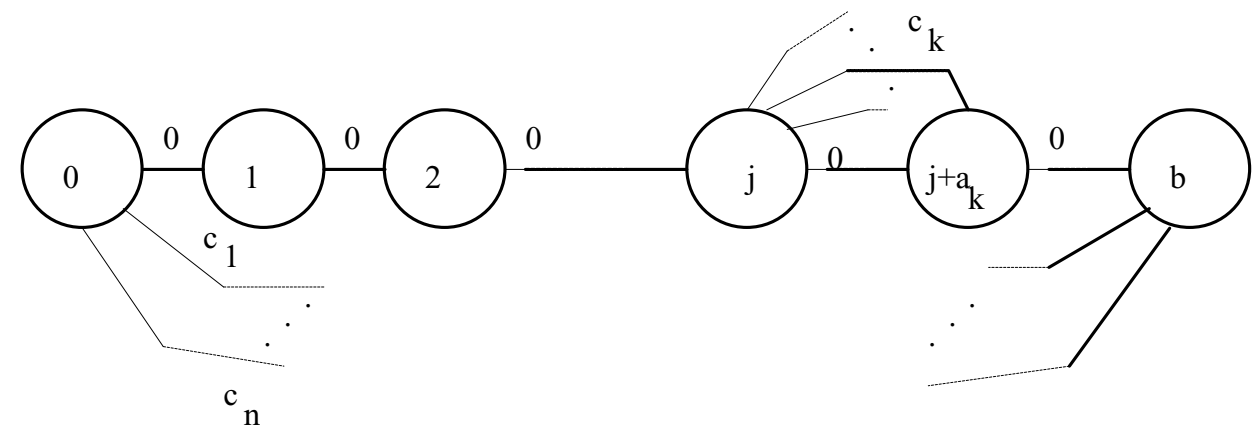

Figure 5

$\mathrm{KP}$ as a longest path problem

With the implementation proposed here many arcs of the graph are not considered, thus avoiding duplicated analysis of equivalent solutions. Additional information of intermediate paths is kept so that information on alternative solutions and not only the best solution are retrieved with increased efficiency. It is important to note that other known algorithms for solving the KKP, based on the approach suggested previously, e.g. Katoh, Ibaraki and Mine [1982], Perko [1986], Sckiscim and Golden [1989] and, more recently, Eppstein [1994], are designed for the 0-1 case. No direct comparison among those methods with the one suggested here can be made, since the corresponding number of varivables $(n)$ increases for the 0-1 case, which, in turn, affects the quantity of arcs in the "knapsack graph".

Note that the computational complexity of finding the K-longest loopless paths from node 0 to node $b$, in a general network with $(b+1)$ nodes using the proposed enumeration scheme is $\mathrm{O}\left((\mathrm{K}+1) \mathrm{b}^{2}\right)$. Exactly the same reasoning can be applied to find the $\mathrm{K}$-shortest loopless paths in a network, hence, with the same computational complexity. Observe that in terms of computational complexity this scheme to solve the K-shortest loopless paths in a network is better than the one suggested by Lawler [1972] which requires $\mathrm{O}\left(\mathrm{Kb}^{3}\right)$.

Acknowledgements: The authors acknowledge Mr. Rodrigo de Castro Penna Franca for the implementation and computational results. This work was partially funded by $\mathrm{CNPq}-$ Conselho Nacional de Desenvolvimento Científico e Tecnológico and FAPESP - Fundação de Amparo à 
Pesquisa do Estado de São Paulo. The authors also thanks for the comments of the anonymous referees that improved the presentation of the paper.

\section{References}

(1) Eppstein, D. (1994) Finding the k Shortest Paths, Proc. 25th IEEE Annual Symposium on Foundation of Computer Science, 154-165.

(2) Gilmore, P.; Gomory, R. (1961) A linear programming approach to the cutting stock problem. Operations Research, 9, 849-859.

(3) Gilmore, P.; Gomory, R. (1963) A linear programming approach to the cutting stock problem - part II. Operations Research, 11, 863-888.

(4) Gilmore, P.; Gomory, R. (1965) Multistage cutting stock problems of two and more dimensions. Operations Research, 14, 1045-1074.

(5) Katoh, N.; Ibaraki, T.; Mine, H. (1982) An efficient algorithm for the shortest simple paths, Networks, 12, 411-448.

(6) Lawler, E.L. (1972) A procedure for computing the k-best solutions to discrete optimization problems and its application to the shortest path problem. Management Science, 18 (7), 401405.

(7) Maculan, N.; Michelon, P.; Plateau, G. (1992) Column-generation in linear programming with bounding variable constraints and its application in integer programming. Pesquisa Operacional, 12 (2), 45-57.

(8) Perko A. (1986) Implementations of algorithms for k shortest loopless paths, Networks, 16, 149-160.

(9) Martello, S.; Toth, P. (1990) Knapsack problems - Algorithms and Computer Implementations. John Wiley \& Sons, Chichester.

(10) Sckiscim C.C. and Golden, B.L. (1989) Solving k-shortest and constrained shortest path problems efficiently, Annals of Operations Research, 20, 249-282.

(11) Soma, N.Y.; Yanasse, H.H.; Zinober, A.S.I.; Harley, P. J. (1992) A pseudopolylogarithmic algorithm for the subset sum problem. CO92 - Combinatorial Optimization Conference, University of Oxford, Oxford, England.

(12) Toth, P. (1980) Dynamic programming algorithms for the zero-one knapsack problem, Computing, 25, 29-45. 
(13) Wolsey, L.A. (1973) Generalized dynamic programming methods in integer programming. Mathematical Programming, 4, 222-232.

(14) Yanasse, H.H.; Soma, N.Y. (1987) A new enumeration scheme for the knapsack problem. Discrete Applied Mathematics, 18, 235-245.

(15) Yanasse, H.H.; Soma, N.Y. (1990) Finding the k-best solutions to a value independent knapsack problem. IFORS XII, Athens, Greece.

\title{
Appendix - The K-best algorithm
}

Given such that

positive integers $b, n, K, a_{i}, i=1,2, \ldots, n$,

$$
n \leq K \text { and } a_{1} \leq a_{2} \leq \ldots \leq a_{n},
$$

Notation

non-negative real numbers $c_{i}, i=1,2, \ldots, n$. If for some $i, a_{i}=a_{i+1}$ then $c_{i} \geq c_{i+1}$.

- Variables in bold indicate vectors;

- $\quad \mathbf{0}$ is a vector with all components equal to 0 .

- $\mathbf{1}$ is a vector with all components equal to 1 .

- // line with comments

\section{The algorithm}

\author{
Main Program \\ Begin-Main \\ // Begin-Initialization \\ Generate matrix $\mathbf{M}_{b \mathrm{X} n}$ \\ $\mathbf{M}(r, s) \leftarrow-1, r=1, \ldots, b$ and $s=1, \ldots, n$ \\ // End-Initialization \\ // Begin-Forward Enumeration \\ // Begin initial ramification \\ For $j=1$ to $n$ do $\mathbf{M}\left(a_{j}, j\right) \leftarrow c_{j}$; \\ // End-initial ramification \\ // Begin-ramification of supernodes \\ For $t=a_{1}$ to $\left(b-a_{1}\right)$ do \\ Begin-For

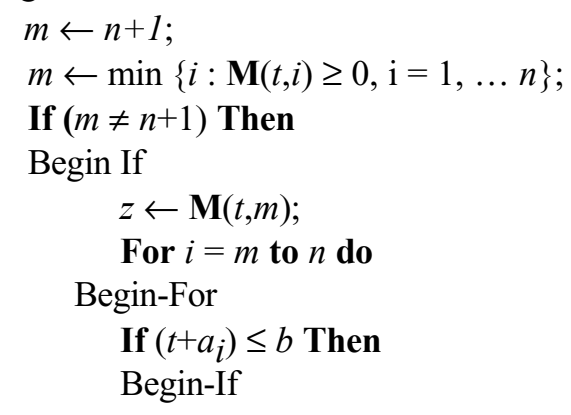




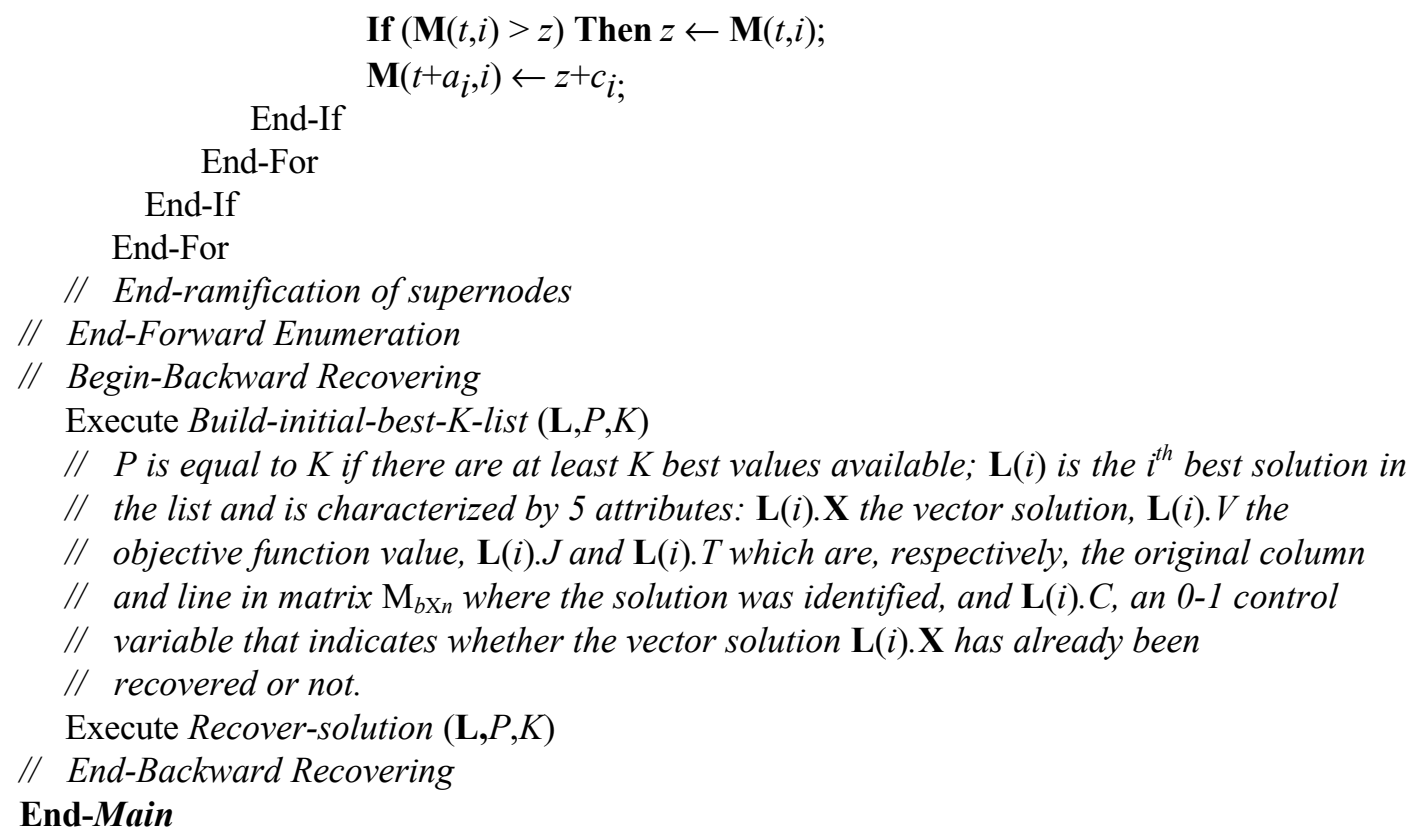




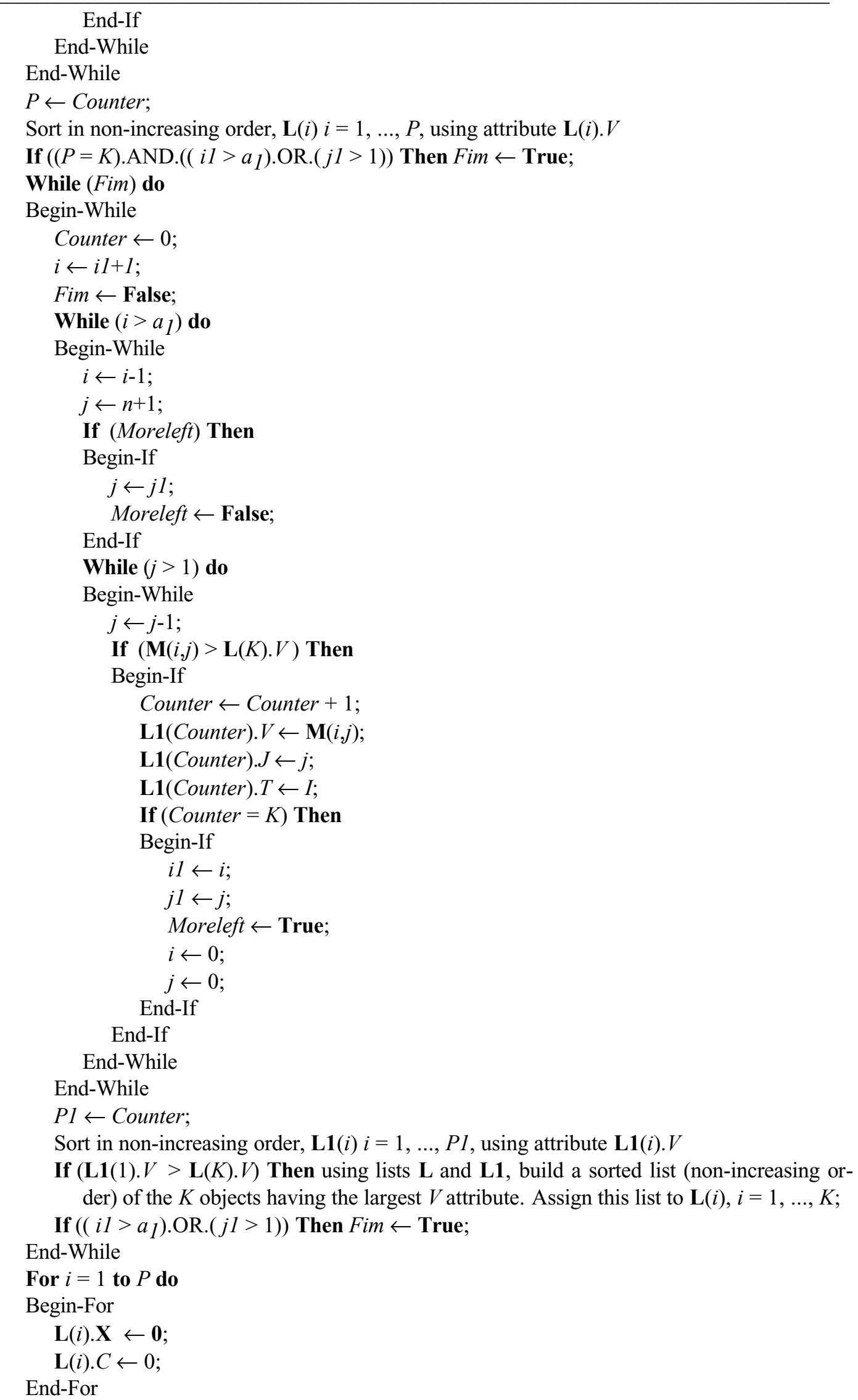


End // Build-initial-best-K-list

Procedure Recover-solution ( $\mathbf{L}, P, K)$

Begin // Recover-solution

$i \leftarrow 0$;

While $(i \leq P)$ do

Begin-While

$$
\begin{aligned}
& i \leftarrow i+1 ; \\
& \text { If }(\mathbf{L}(i) . C=0) \text { Then } \\
& \text { Begin-If }
\end{aligned}
$$

$\mathbf{A U X L} \leftarrow \mathbf{L}(i)$

Call Backtracking (L, AUXL, $i, P, K)$;

$\mathbf{L}(i) \leftarrow \mathbf{A U X L}$;

$$
\text { End-If }
$$

End-While

End // Recover-solution

Procedure Backtracking (L,AUXL, $i, P, K$ )

Begin // Backtracking

$t \leftarrow$ AUXL.T;

$j \leftarrow$ AUXL. $J$;

$z \leftarrow$ AUXL. $V$;

zcum $\leftarrow 0$;

While $(t>0)$ do

Begin-While

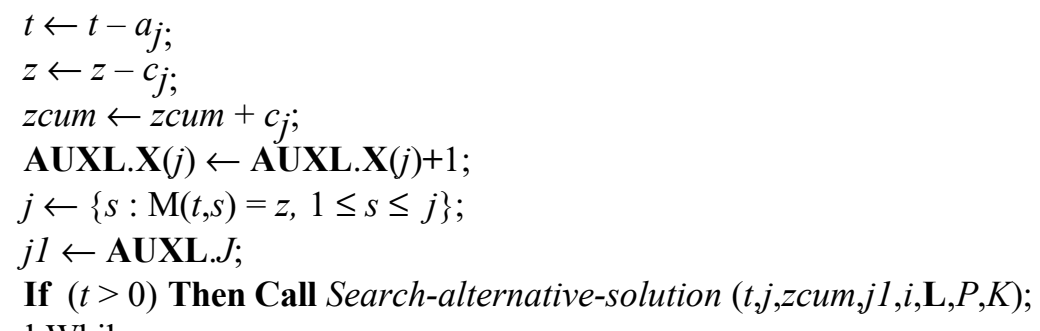

End-While

AUXL. $C \leftarrow 1$;

End // Backtracking

Procedure Search-alternative-solution $(t, j, z c u m, j 1, i, \mathbf{L}, P, K)$

Begin // Search-alternative-solution

For $s=1$ to $j 1$ do

Begin-For

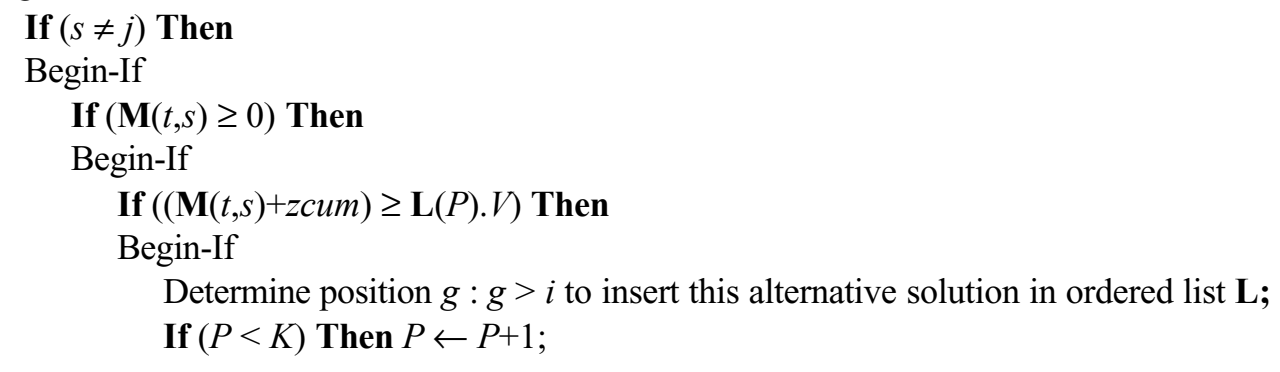




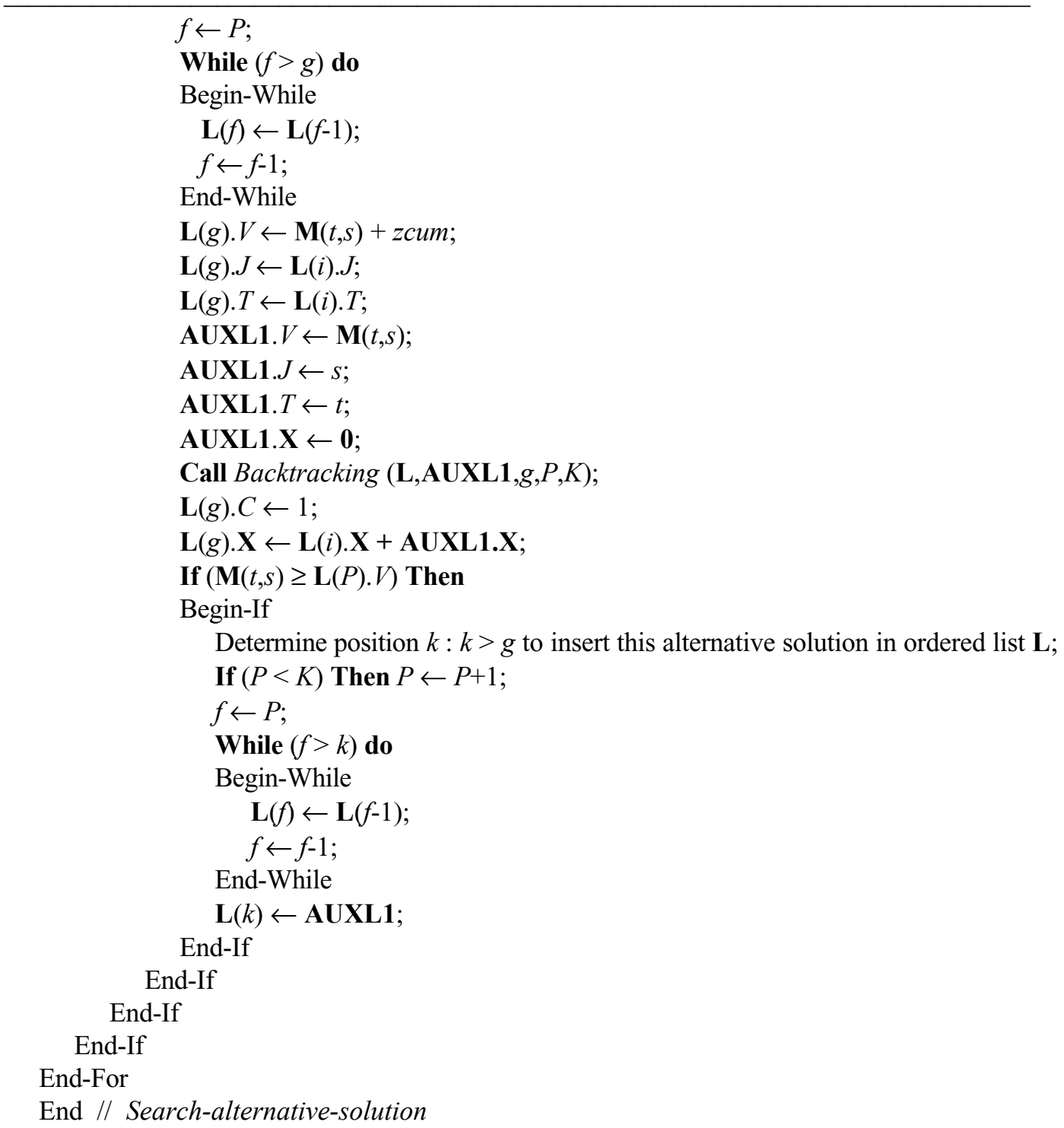

